\title{
Phosphorylation Promotes Neurotoxicity in a Caenorhabditis elegans Model of TDP-43 Proteinopathy
}

\author{
Nicole F. Liachko, ${ }^{1,2 *}$ Chris R. Guthrie, ${ }^{1 *}$ and Brian C. Kraemer ${ }^{1,3}$ \\ ${ }^{1}$ Geriatrics Research Education and Clinical Center, Veterans Affairs Puget Sound Health Care System, Seattle, Washington 98108, and ${ }^{2}$ Department of \\ Pathology and ${ }^{3}$ Division of Gerontology and Geriatric Medicine, Department of Medicine, University of Washington, Seattle, Washington 98104
}

Neurodegenerative disorders characterized by neuronal and glial lesions containing aggregated pathological TDP-43 protein in the cytoplasm, nucleus, or neurites are collectively referred to as TDP-43 proteinopathies. Lesions containing aggregated TDP-43 protein are a hallmark of amyotrophic lateral sclerosis (ALS) and frontotemporal lobar degeneration with ubiquitinated inclusions (FTLD-U). In addition, mutations in human TDP-43 cause ALS. We have developed a Caenorhabditis elegans model of TDP-43 proteinopathies to study the cellular, molecular, and genetic underpinnings of TDP-43-mediated neurotoxicity. Expression of normal human TDP-43 in all $C$. elegans neurons causes moderate motor defects, whereas ALS-mutant G290A, A315T, or M337V TDP-43 transgenes cause severe motor dysfunction. The model recapitulates some characteristic features of ALS and FTLD-U including age-induced decline in motor function, decreased life span, and degeneration of motor neurons accompanied by hyperphosphorylation, truncation, and ubiquitination of TDP-43 protein that accumulates in detergent-insoluble protein deposits. In C. elegans, TDP-43 neurotoxicity is independent of activity of the cell death caspase CED-3. Furthermore, phosphorylation of TDP-43 at serine residues 409/410 drives mutant TDP-43 toxicity. This model provides a tractable system for additional dissection of the cellular and molecular mechanisms underlying TDP-43 neuropathology.

\section{Introduction}

TDP-43 proteinopathies include a variety of neurodegenerative diseases characterized by neuronal and glial lesions containing insoluble aggregated TDP-43. The most prevalent TDP-43 proteinopathies are amyotrophic lateral sclerosis (ALS) and frontotemporal lobar degeneration with ubiquitinated TDP-43 inclusions (FTLD-TDP). ALS is a severe neurodegenerative disease affecting $\sim 5$ per 100,000 people and is characterized by degeneration of motor neurons in the brain and spinal cord, resulting in fasciculations and loss of voluntary movement (for review, see Wijesekera and Leigh, 2009). ALS progresses rapidly, with an average survival of only 3 years after diagnosis. Nearly 95\% of ALS cases have pathological features including ubiquitinated, hyperphosphorylated inclusions of TDP-43 in neurons and glial cells (Arai et al., 2006; Neumann et al., 2006). These inclusions are a hallmark of FTLD-TDP, which manifests as degeneration of the frontal and temporal lobes, resulting in behav-

\footnotetext{
Received June 8, 2010; revised Aug. 31, 2010; accepted Oct. 1, 2010.

This work was supported by a Department of Veterans Affairs Merit Review Grant (B.C.K.) and by National Institute of Neurological Disorders and Stroke Grant R01 NS064131 (B.C.K.). N.F.L. was supported by National Institute on Aging Training Grant AG 000057-31. We thank Dr. James H. Thomas for advice regarding C. elegans genetics and strains. We thank Drs. Gerard Schellenberg and Chang-En Yu for helpful discussion regarding human genetics and TDP-43. We thank the C. elegans Genetics Center for providing strains. We thank Elaine Loomis, Leo Anderson, and Tobin Martin for outstanding technical assistance. We thank Andrew Fire for providing C. elegans expression vectors, Yishi Jin for strain CZ1200, James Rand for plasmid RM\#509p, and the Developmental Studies Hybridoma Bank (National Institute of Child Health and Human Development) for the $\beta$-tubulin antibody E7.

*N.F.L. and C.R.G. contributed equally to this work.

Correspondence should be addressed to Brian C. Kraemer, Seattle Veterans Affairs Puget Sound Health Care System, S182, 1660 South Columbian Way, Seattle, WA 98108. E-mail: kraemerb@u.washington.edu.

DOI:10.1523/JNEUROSCI.2911-10.2010

Copyright $\odot 2010$ the authors $\quad$ 0270-6474/10/3016208-12\$15.00/0
}

ioral and cognitive abnormalities and dementia (Kertesz, 2009). Although different populations of neurons are destroyed, both ALS and FTLD-TDP affect aging adults and display progressive neurodegeneration resulting in severe disability and shortened life span. An increasing number of mutations in the gene coding for TDP-43, TARDBP, have been identified in both familial and sporadic ALS (Pesiridis et al., 2009) as well as frontotemporal lobar degeneration with ubiquitinated inclusions (FTLD-U) (Benajiba et al., 2009).

TDP-43 is known to affect transcription, pre-mRNA splicing, and mRNA stability (for review, see Buratti and Baralle, 2008; Wang et al., 2008). The protein contains two RNAbinding motifs capable of binding to both DNA and RNA (Ou et al., 1995; Buratti et al., 2001), a glycine-rich domain necessary for CFTR exon-9 skipping and interaction with hnRNPs (heterogeneous nuclear ribonucleic proteins) (Buratti et al., 2005), and nuclear localization and export signals (Winton et al., 2008). Interestingly, the majority of TARDBP mutations identified in ALS reside in exon 6, encoding the TDP-43 C-terminal glycine-rich domain.

Ubiquitinated inclusions of TDP-43 isolated from brain and spinal cord tissue contain aggregated full-length and truncated C-terminal TDP-43 fragments (Igaz et al., 2008). TDP-43 has several putative caspase cleavage sites, and recent work in cell culture suggests TDP-43 cleaved by caspase contributes to neurotoxicity in ALS (Zhang et al., 2007, 2009). In addition, pathological TDP-43 is hyperphosphorylated, and this phosphorylation can be used to distinguish pathological TDP-43 from normal TDP-43 (Inukai et al., 2008; Neumann et al., 2009). It is unknown whether ubiquitination, truncation, or 
hyperphosphorylation of TDP-43 contribute to neurotoxicity or result as a consequence of neurodegeneration.

To dissect the TDP-43 pathogenic mechanisms, it is necessary to develop in vivo model systems representing features of both wild-type (WT) and mutant TDP-43. We have generated a model of human TDP-43 pathology in Caenorhabditis elegans, which displays molecular and cellular characteristics of TDP-43 proteinopathies including ALS and FTLD-U. This model system will allow additional characterization of TDP-43 neurotoxicity and provide a system to rapidly test different neuroprotective strategies and to explore possible mechanisms of TDP-43 neurotoxicity.

\section{Materials and Methods}

Plasmids. To generate transgenes expressing human TDP-43 driven by a pan-neuronal snb-1 promoter, a cDNA clone encoding the major wildtype neuronal isoform of TDP-43 (NM_007375) was obtained from Open Biosystems. This wild-type TDP-43 cDNA was used in sitedirected mutagenesis reactions (Stratagene QuikChange reagents) to generate familial ALS-mutant cDNAs carrying the G290A (Van Deerlin et al., 2008), A315T (Gitcho et al., 2008; Sreedharan et al., 2008), or M337V (Sreedharan et al., 2008) missense mutations in TDP-43. The wild-type and each mutant TDP-43 cDNA served as templates for highfidelity PCR (Stratagene; Pfu Hotstart) with KpnI and SacI restriction sites introduced at the $5^{\prime}$ and $3^{\prime}$ end of the cDNA, respectively. KpnI and SacI fragments were inserted into vector $\mathrm{P}_{\text {snb-1 }}$ cut with KpnI and SacI to generate transgene constructs $\mathrm{P}_{\text {snb- } 1}:: T D P-43$ (WT), $\mathrm{P}_{\text {snb-1 }}:: T D P-43$ (G290A), $\mathrm{P}_{\text {snb- } 1}::$ TDP-43 (A315T), and $\mathrm{P}_{\text {snb- } 1}::$ TDP-43 (M337V). The parental $\mathrm{P}_{\text {snb-1 }}$ vector was constructed by inserting the $s n b-1$ promoter sequences into the HindIII and BamHI sites of pPD49.26 (a generous gift from Dr. A. Fire, Stanford University, Palo Alto, CA).

Transgenics and strains. N2 (Bristol) was used as wild-type C. elegans and maintained as previously described (Brenner, 1974). The marker transgene $\mathrm{P}_{\text {myo- }}::$ dsRED was used at a concentration of $10 \mathrm{ng} / \mu \mathrm{l}$ as a coinjection marker as previously described (Guthrie et al., 2009). The $\mathrm{P}_{\text {snb-1 }}:$ TDP-43 (WT), $\mathrm{P}_{\text {snb-1 }}:$ TDP-43 (G290A), $\mathrm{P}_{\text {snb-1 }}:$ TDP-43 (A315T), $\mathrm{P}_{\text {snb-1 }}:: T D P-43(\mathrm{M} 337 \mathrm{~V})$, and $\mathrm{P}_{\text {snb- } 1}:$ GFP (plasmid RM\#509p; a generous gift from Dr. J. Rand, Oklahoma Medical Research Foundation, Oklahoma City, OK) transgenes described above were microinjected into $\mathrm{N} 2$ at a concentration of $160-180 \mathrm{ng} / \mu \mathrm{l}$ as previously described (Kraemer et al., 2003) to produce worms with TDP-43 or green fluorescent protein (GFP) and dsRED fluorescent protein (RFP) transgenes as extrachromosomal arrays. Transgenes were integrated by exposing animals to a dose of 3200-3600 rad gamma rays and integrated strains were backcrossed to $\mathrm{N} 2$ at least twice.

Strain CZ1200 (a generous gift from Dr. Y. Jin, University of California, San Franciso, CA) has an integrated $\mathrm{P}_{\text {unc-25 }}$ : GFP transgene expressed in GABAergic neurons that clearly marks the cell bodies and axons of ventral cord VD and DD type inhibitory motor neurons (Cinar et al., 2005). Strains MT1522 ced-3(n717) and MT2547 ced-4(n1162) were obtained from the C. elegans Genetics Center. All TDP-43 transgenic strains described here contain integrated transgenes carrying either wild-type or mutant TDP- 43 coding cDNAs and a $\mathrm{P}_{\text {myo- } 2}:$ dsRED coinjection marker. CK405 and CK410 carry $\mathrm{P}_{\text {snb-1 }}:$ TDP-43 (WT) expressed at higher and lower levels, respectively. CK422, CK423, and CK426 carry $\mathrm{P}_{\text {snb-1 }}::$ TDP-43 (G290A), $\mathrm{P}_{\text {snb- } 1}:: T D P-43$ (M337V), and $\mathrm{P}_{\text {snb- } 1}:: T D P-43$ (A315T) mutant TDP-43 transgenes, respectively. These strains were crossed singly or in combination with CZ1200, MT1522, or MT2547 strains to generate ced-3 and ced-4 mutants with TDP-43 transgenes and/or doubletransgenic animals carrying the $\mathrm{P}_{\text {unc-25 }}:$ GFP GABAergic marker transgene. CK505, CK506, and CK507 are independently isolated lines carrying $\mathrm{P}_{\text {snb-1 }}::$ TDP-43 (M337V, S409A/S410A), and CK510 and CK511 are independently isolated lines carrying $\mathrm{P}_{\text {snb- }}:$ :TDP-43 (G290A, S409A/S410A).

Radial locomotion assay. Radial locomotion assays were used as described except longer time points were used (Robatzek and Thomas, 2000). The locomotion of control and transgenic worms was assayed by picking 10-20 L4 or adult worms onto $150 \mathrm{~mm}$ nematode growth medium (NGM) agar plates spread with a uniform bacterial lawn. Worm locations were then recorded at the indicated time points, and linear distances from the starting position were measured. Statistical analysis was performed using GraphPad Prism software.

Life span assay. Life span assays were modified from those described by Liachko et al. (2009). In brief, worms were grown at $20^{\circ} \mathrm{C}$ after a short (4-6 h) egg lay to L4 stage on NGM plates seeded with Escherichia coli OP50, and then transferred onto seeded NGM plates with added FUDR (5-fluoro-2'-deoxyuridine) $(0.05 \mathrm{mg} / \mathrm{ml})$ to inhibit growth of progeny. Worms were scored every $2-3 \mathrm{~d}$ by gentle touching with a platinum wire. Failure to respond to touch was scored as dead. Statistical analysis was performed using GraphPad Prism software.

Protein extraction. Total protein fractions were obtained by lysing worms in RAB high salt buffer by homogenization as previously described (Guthrie et al., 2009). For extraction of insoluble TDP-43 protein aggregates, fractions were obtained using methods previously described for analysis of FTLD-U and ALS brain samples (Neumann et al., 2006). Briefly, packed worms pellets were resuspended in twice the amount (w/v) of low-salt buffer (LS) (10 mm Tris, 5 mm EDTA, 10\% sucrose, $\mathrm{pH}$ 7.5). Worms were completely lysed by sonication and homogenates centrifuged at $25,000 \times g$ for $30 \mathrm{~min}$. The supernatant constitutes the LS fraction. The pellet was reextracted with nonionic detergent-containing buffer (TX) (10 mm Tris, 5 mm EDTA, 1\% Triton X-100, 10\% sucrose, $\mathrm{pH} 7.5$ ), centrifuged $20 \mathrm{~min}$ at $180,000 \times g$, with the supernatant constituting the TX fraction. The resulting pellet was extracted with ionic detergent containing buffer (SARK) (10 mM Tris, $5 \mathrm{~mm}$ EDTA, $1 \%$ Sarkosyl, $10 \%$ sucrose, pH 7.5) and $180,000 \times g$ for $20 \mathrm{~min}$. The supernatant is the SARK fraction. The final detergent-insoluble pellet was solubilized in urea containing buffer (UREA) [30 mM Tris, $7 \mathrm{~m}$ urea, $2 \mathrm{M}$ thiourea, 4\% CHAPS (3-[(3-cholamidopropyl)dimethylammonio]-1propanesulfonate), $\mathrm{pH}$ 8.5]. All buffers contained Complete protease inhibitor mixture (Roche) and $0.5 \mathrm{~mm}$ phenylmethylsulfonylfluoride to block proteolysis.

Immunoblotting. Equivalent worm lysate fractions were loaded and resolved on precast $4-15 \%$ gradient SDS-PAGE gels and transferred to polyvinylidene difluoride membrane as recommended by the manufacturer (Bio-Rad). On immunoblots, human TDP-43 was detected with a commercially available monoclonal antibody ab57105 (Abcam) directed against human TDP-43 amino acids 1-261. TDP-43 phosphorylated at S409/S410 was detected by a monoclonal antibody called anti-phosphoTDP-43 (pS409/410) available from Cosmobio (catalog \#TIP-PTDM01). C. elegans $\beta$-tubulin levels were measured using monoclonal antibody E7 as a loading control as previously described (Kraemer and Schellenberg, 2007; Guthrie et al., 2009). HRP-labeled goat anti-mouse IgG was the secondary antibody (GE Healthcare) and used at a dilution of 1:4000. Dilutions were as follows: 1:7500 for ab57105, 1:1000 for pS409/ 410, and 1:10,000 for E7.

Immunocytochemistry. Worms were fixed in $1 \%$ formaldehyde solution and permeabilized by freeze cracking as described previously (Crittenden and Kimble, 1999). Fixed and permeabilized animals were counterstained with $300 \mathrm{~nm} 4^{\prime}$,6-diamidino-2-phenylindole (DAPI) nuclear stain in PBS. Fixed whole animals were stained with anti-TDP-43 monoclonal antibody at a dilution of 1:1000 or anti-phospho-TDP-43 (pS409/410). Alexa 488-conjugated anti-mouse antibody (Invitrogen) was used as the secondary antibody at a dilution of 1:1000. Images were acquired and analyzed as described below.

Live-mount fluorescence microscopy. Live worms were mounted as described previously (Crittenden and Kimble, 1999). Imaging of live worms was done on a Nikon Eclipse TE300 epifluorescent microscope. Images were acquired using a Photometrics SenSys cooled CCD camera and IPLab image acquisition software (BD Biosciences Bioimaging). Images were deconvolved using MicroTome deconvolution software (BD Biosciences Bioimaging).

Neurodegeneration assays. Timed egg lays were arranged to produce synchronized populations 8,24 , and $48 \mathrm{~h}$ of age. Live worms were placed on a $3 \%$ agarose pad containing $0.01 \%$ sodium azide to immobilize the worms. Worms were imaged under fluorescence microscopy and scored for number of GABAergic neurons, gaps in the ventral nerve cord, and gaps in the dorsal nerve cord. Data were analyzed using GraphPad Prism software. 


\section{Results}

\section{Expression of human TDP-43 causes} motor defects

To test whether wild-type human TDP-43 is capable of producing pathological phenotypes in C. elegans, we generated worm lines expressing the most abundant CNS isoform of human TDP-43 in all C. elegans neurons driven by the synaptobrevin $(s n b-1)$ promoter. We observed that panneuronal expression of human TDP-43 in C. elegans causes defects in motor function as indicated by an uncoordinated movement (Unc) phenotype. By visual inspection, the Unc phenotype varied in severity from line to line, but Unc severity did not correlate with TDP-43 protein expression levels (Fig. 1D). For animals expressing wild-type human TDP-43, this Unc phenotype is characterized by lethargy, flattened sinusoidal waveform, and reduced locomotion in response to stimuli (see supplemental Movies WT-high and WT-low, available at www.jneurosci. org as supplemental material).

\section{ALS mutations worsen TDP-43 movement abnormalities}

An increasing number of mutations in TARDBP, the gene encoding TDP-43, have been identified in cases of sporadic and familial ALS. The mutations identified in familial ALS are especially informative, as the pathogenic mutations in TDP-43 segregate with the disorder in these ALS-affected families, demonstrating that abnormal TDP-43 can cause degeneration of motor neurons. To test the effect of familial ALS mutations on the function and pathology of TDP-43, we chose three mutations to test in C. elegans. Single amino acid changes in TDP-43 at residues G290A (Van Deerlin et al., 2008), A315T (Gitcho et al., 2008; Kabashi et al., 2008), and M337V (Rutherford et al., 2008; Sreedharan et al., 2008) have been identified in multiple cases of familial ALS. All three mutations fall in the glycine-rich C-terminal portion of TDP43 , where the majority of TDP-43 mutations have been identified (Fig. $1 A$ ). We expressed mutant TDP-43 using the $s n b-1$ pan-neuronal promoter, and compared multiple lines of integrated transgenes. $C$. elegans expressing any of the mutant forms of TDP-43 showed significantly impaired movement compared with animals expressing wild-type TDP-43 (Fig. $1 B, C$ ). In particular, adult animals expressing ALSmutant TDP-43 were characterized by partial or complete paralysis, slowed movement, exaggerated wave form, unresponsiveness, and coiling (Fig. 1B,C; supplemental Movies A315T, G290A, and $\mathrm{M} 337 \mathrm{~V}$, available at www.jneurosci.org as supplemental material).
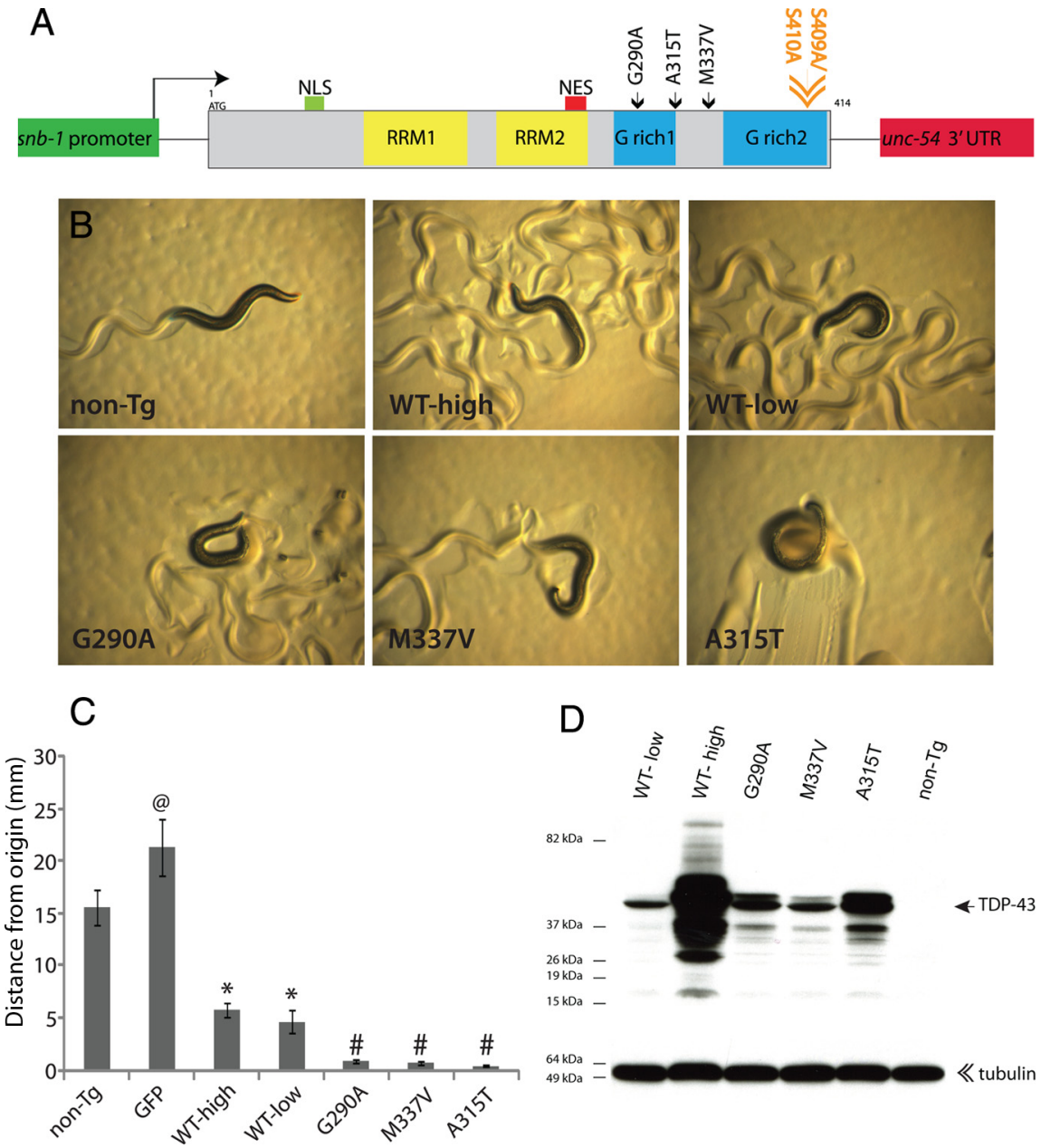

$\mathrm{D}$

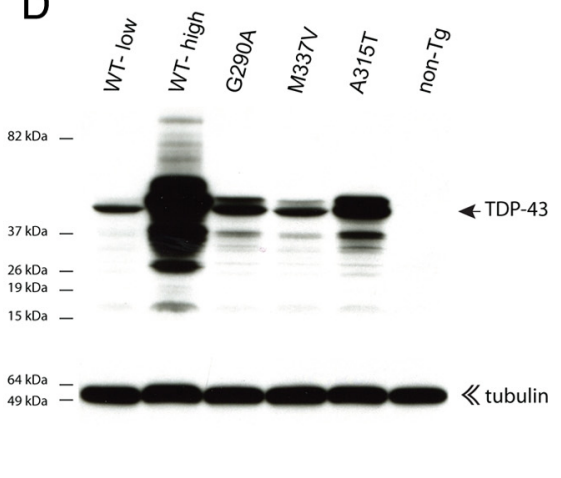

Figure 1. Human TDP-43 causes motor defects in C. elegans. A, Schematic of TDP-43 construct injected into wild-type C. elegans. The most abundantly expressed TDP-43 CDNA is driven by the pan-neuronal promoter snb-1. TDP-43 contains nuclear localization and export signals (NLS and NES, respectively), two RNA recognition motifs (RRM1 and RRM2), and (-terminal glycine-rich regions. The familial ALS mutations introduced into wild-type TDP-43 are marked with black arrows. Serines 409/410, which are mutated to alanine in the M337V/AA and G290A/AA strains, are marked with a double-headed arrow. The 3' -untranslated region (3' -UTR) of unc -54 is included to facilitate expression of the TDP-43 protein in worms. $\boldsymbol{B}$, Worms expressing wild-type or mutant TDP-43 display severe movement defects compared with nontransgenic controls (non-Tg). Worms were grown to day 1 of adulthood, placed on seeded NGM plates, and photographed after $<1 \mathrm{~h}$ of movement. Nontransgenic (non- $\mathrm{Tg}$ ) worms move normally across a bacterial lawn, as evidenced by a smooth waveform track and sinusoidal body position. Worms expressing wild-type TDP-43 are severely uncoordinated, with exaggerated wave form and coiling, but are capable of moving short distances. Expression of mutant TDP-43 causes partial or complete paralysis, slowed movement, exaggerated wave form, unresponsiveness, and coiling, with little or no displacement from the starting position. C, Worms expressing wild-type TDP-43 travel significantly shorter distances than non-Tg controls or transgenic controls expressing pan-neuronal GFP, and expression of mutant TDP-43 further impairs travel. In contrast, GFP transgenic control worms exhibited slightly increased locomotion compared with non-Tg controls. Stage synchronized $\mathrm{L} 4$ worms were placed in the center of 150 -mm-diameter NGM plates covered with a uniform bacterial lawn and allowed to move freely for $30 \mathrm{~min}$. The linear distance traveled from the starting location was then recorded after $30 \mathrm{~min}$. Results shown combine data from multiple experiments. Error bars represent SEM. $N=55$, non-Tg; 43, GFP; 60, WT-high; 42, WT-low; 32, G290A; $58, \mathrm{M} 337 \mathrm{~V} ; 48, \mathrm{A315T} .{ }^{{ }^{\circledR}} p<0.01$ versus non-Tg; ${ }^{*} p<0.001$ versus non-Tg; ${ }^{{ }} p<0.05$ versus WT-high. Statistical significance was determined using one-way ANOVA with Tukey's multiple-comparison test. $\boldsymbol{D}$, TDP-43 total protein levels from worm strains expressing wild-type or mutant TDP-43, as indicated. Nontransgenic (non-Tg) worms showed no reactivity to the pan-TDP-43 antibody. The single arrow represents the major full-length isoform of TDP- 43 at $\sim 45 \mathrm{kDa}$ in size. Multiple bands present in TDP-43 transgenic lines indicate TDP-43 truncation products, alternate start sites, or cleavage products of full-length TDP-43. The double arrowhead indicates tubulin, used as a loading control.

C. elegans contains one homolog of TDP-43, called TDP-1. This protein has $39 \%$ primary sequence identity to human TDP-43. The strongest homology resides in the $\mathrm{N}$ terminus and RRM domains, whereas TDP-1 lacks the C-terminal glycine-rich domain entirely. Both $t d p-1$ (ok803) and $t d p-1$ (ok781) deletion strains have no obvious abnormalities in reproduction, development, or movement (data not shown), although they have somewhat reduced locomo- 


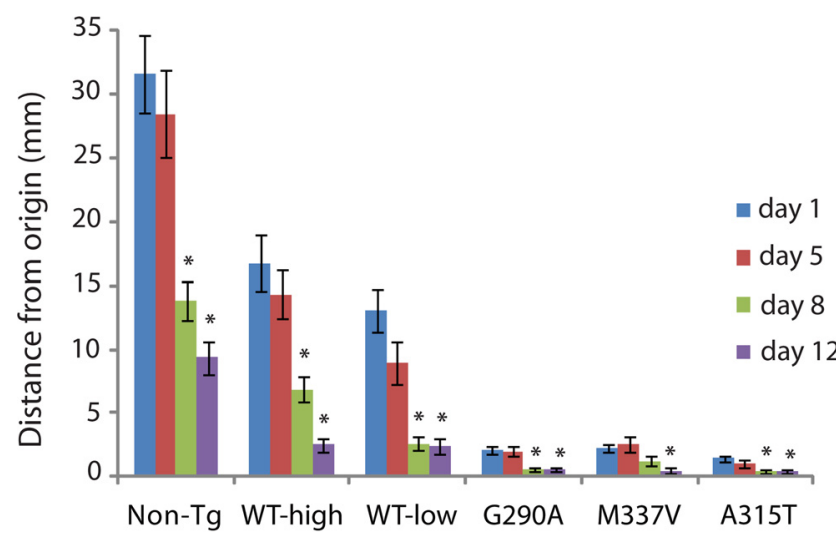

Figure 2. TDP-43 increases aging-related motor decline. TDP-43-dependent defects in locomotion increase over time. Worms were allowed to develop to adulthood, and radial locomotion was measured as described in Figure 1. Locomotion was tested on days 1, 5, 8, and 12 of adulthood, with time points chosen to span the majority of normal worm life span. Nontransgenic and wild-type TDP-43 transgenic lines show decreased dispersal as worms age, with significant movement decline demonstrated by day 8 of adulthood and with TDP-43 worms approaching complete paralysis by day 12 . Mutant TDP-43 lines also demonstrated an additional decrease in movement beyond the severe impairment present at day 1 of adulthood as worms aged. Error bars represent SEM. $N>25$ for all strains tested at each time point. Statistical significance was analyzed using one-way ANOVA with Tukey's multiple-comparison test. ${ }^{*} p<0.05$ versus day 1 of adulthood for each respective strain.

tion relative to wild-type worms (supplemental Fig. S3A, available at www.jneurosci.org as supplemental material). To test whether ALSmutant TDP-43 interacts with $t d p-1$ to cause severe locomotion defects, we generated strains carrying a knock-out of $t d p-1$ with the mutant TDP-43 transgene. Loss of $t d p-1$ does not alter the phenotype of lines carrying human TDP-43 transgenes demonstrating endogenous $t d p-1$ does not participate in TDP-43 neurotoxicity in this model.

\section{Aging exacerbates TDP-43 phenotypes}

Both ALS and FTLD-U result in shortened life span. Therefore, we asked whether wild-type or mutant human TDP-43 transgenes affect the life span of C. elegans (supplemental Table S1, available at www.jneurosci.org as supplemental material). All TDP-43-expressing worms exhibit shortened life span (supplemental Fig. S2, available at www.jneurosci.org as supplemental material). Since both ALS and FTLD-U are adult-onset diseases, and ALS exhibits progressively worsening motor dysfunction, we measured the severity of locomotion defects observed in wildtype and mutant TDP-43 transgenic worms during adult aging. Using a synchronized population of worms, we quantified radial locomotion at several time points throughout adulthood (Fig. 2). We found that all TDP-43-expressing strains exhibited a decline in motor function over time culminating in partial or complete paralysis. Specifically, the locomotion of worms expressing wildtype TDP-43 declined significantly by day 8 of adulthood, with older worms displaying severely impaired movement. As young adults, worms expressing G290A, M337V, or A315T mutant TDP-43 had significantly greater impairment of movement than worms expressing wild-type TDP-43, and showed nearly complete paralysis by day 8 of adulthood. These declines in motility are distinguished from normal worm aging-related declines in motility (for review, see Collins et al., 2008). Normal worms do not become paralyzed except at extremely advanced ages, typically $\sim 18-20 \mathrm{~d}$ as seen in nontransgenic controls (non-Tg). Non-Tg animals exhibit fast body movement until after day 8 (Huang et al., 2004). In contrast, wild-type TDP-43-expressing lines lose fast body movement after day 5 of adulthood, an age less than one-half of mean life span of TDP-43 transgenic animals (supplemental Table S1, available at www.jneurosci.org as supplemental material); mutant TDP-43-expressing lines never display fast body movement even as young adults. These findings suggest aging contributes strongly to motor dysfunction in wildtype TDP-43-expressing lines. But age has lesser significance in TDP-43 mutant lines, because they are so severely impaired by early adulthood.

\section{Human TDP-43 protein accumulates in the nucleus of $C$. elegans neurons}

To explore the distribution of TDP-43, we examined antihuman TDP-43 antibody staining in fixed, permeabilized, adult-stage TDP-43 transgenic worms. As expected, we observed prominent TDP-43 staining in many C. elegans neurons of the head and ventral nerve cord, as dictated by the pan-neuronal $s n b-1$ promoter driving human TDP-43 transgene expression. Neurons were identified by characteristic neuronal morphology under Normarski/differential interference contrast optics (data not shown). We further investigated the subcellular localization of TDP-43 in the TDP-43 WT-high, WT-low, G290A, M337V, and A315T strains at all stages by immunocytochemistry (Fig. 3; supplemental Fig. S2, available at www.jneurosci.org as supplemental material). Epifluorescent microscopy of the immunofluorescent anti-TDP-43 stain showed both wild-type (Fig. $3 A, B, E$; supplemental Fig. S2, available at www.jneurosci.org as supplemental material) and mutant forms of TDP-43 (Fig. 3C,D, F; supplemental Fig. S2, available at www.jneurosci.org as supplemental material) are localized to subdomains within the nucleus of $C$. elegans neurons. These subdomains share a similar gross appearance with the neuronal nuclear inclusions seen in cases of TDP-43 proteinopathy (Neumann, 2009). Few differences are apparent when comparing staining among wild-type TDP-43 and G290A, A315T and M337V mutant lines (supplemental Fig. S2, available at www.jneurosci.org as supplemental material). TDP-43 staining is not detectable in the neuronal cytoplasm or neurites of these lines. We confirmed TDP-43 subcellular localization in living animals by constructing a TDP-43 G290A::GFP fusion protein-encoding transgene driven by the $s n b-1$ promoter. Examination of live mounts revealed GFP fluorescence in the nucleus of neuron cell bodies consistent with the staining pattern seen for untagged human TDP-43 (data not shown).

\section{ALS-mutant but not wild-type TDP-43 protein causes degeneration of GABAergic motor neurons}

Because worms expressing human TDP-43 exhibit motor defects, we examined the structural integrity of GABAergic D-type motor neurons and their processes in transgenic animals expressing TDP-43 (Fig. 4A, drawing) (Jorgensen, 2005). To visualize neurons in living animals, we used the $\mathrm{P}_{\text {unc-25 }}:$ GFP reporter transgene driving expression of GFP in all 19 GABAergic motor neurons (Cinar et al., 2005). In a strain carrying the GFP reporter alone (CZ1200), both the dorsal and ventral nerve cords are continuous and contain the normal complement of 19 inhibitory motor neurons (13 ventral D-type and 6 dorsal D-type GABAergic neurons) (Fig. 4A,B, Table 1). All three transgenic worm strains expressing mutant TDP-43 (G290A, M337V, or A315T) exhibited profound degeneration of the GABAergic motor neuron network (Fig. 4E-J; Table 1, top). This deterioration includes loss of neuron cell bodies and the extension of gaps in neurites of both the ventral and dorsal nerve cords. Although axonal abnormalities were evident beginning by larval stage L1 (day 1 after 
hatching) (supplemental Fig. S4, available at www.jneurosci.org as supplemental material), frank loss of neuronal cell bodies did not begin until between the L2 and L3 larval stages. Interestingly, worms expressing the wild-type TDP-43 transgene showed no significant neurodegeneration compared with worms expressing the reporter alone (Fig. 4C,D; Table 1, top). This is consistent with the more severe motor defects exhibited by worms expressing mutant TDP-43 compared with wild-type TDP-43 (Fig. 1). To test whether additional neuronal subtypes are affected by wild-type or mutant TDP-43, we visualized all dopaminergic neurons in C. elegans by driving expression of GFP with the dopaminergic neuron-specific promoter dat-1 (Lakso et al., 2003). Consistent with the pattern of degeneration of GABAergic neurons shown above, all dopaminergic neurons remained intact in the presence of wild-type TDP-43, but ADE and CEP classes of dopaminergic neurons exhibited axonal loss and frank neurodegeneration in the presence of ALS-mutant TDP-43 (data not shown).

\section{TDP-43 forms insoluble aggregates} One of the characteristic biochemical features of FTLD-U and ALS is the formation of detergent-insoluble TDP-43 deposits and abnormal N-terminally truncated TDP-43 species (Neumann et al., 2006) (for review, see Forman et al., 2007). To investigate whether expression of TDP-43 causes the formation of detergent-insoluble deposits, we analyzed the solubility of TDP-43 protein extracted from transgenic animals. Using the methods used to initially extract TDP-43 from ubiquitinated inclusions (Neumann et al., 2006), we treated TDP-43 transgenic worms to a regimen of sequential extractions with buffers of increasing solubilizing strengths. Fractions from the sequential extraction were analyzed by immunoblotting with TDP-43-specific antibodies to determine solubility of TDP-43 protein (Fig. 5). As seen in Figure $1 D$, the levels of TDP-43 vary from line to line. We observe that detergent-insoluble (urea fraction) TDP-43 is present in all lines. However, the levels of insoluble TDP-43 vary dramatically; the highest levels of insoluble TDP-43 are observed in the TDP-43 WT-high line. Multiple species of TDP-43 are seen, including highmolecular-weight aggregates and truncated species. Interestingly, only a small fraction of the total wild-type TDP-43 protein is soluble in the mildest low-salt extraction, whereas the majority is detergent insoluble. However, the protein levels of ALS-causing mutant versions of TDP-43 are distributed more equally between the low-salt, nonionic detergent, ionic detergent, and detergent-insoluble fractions.
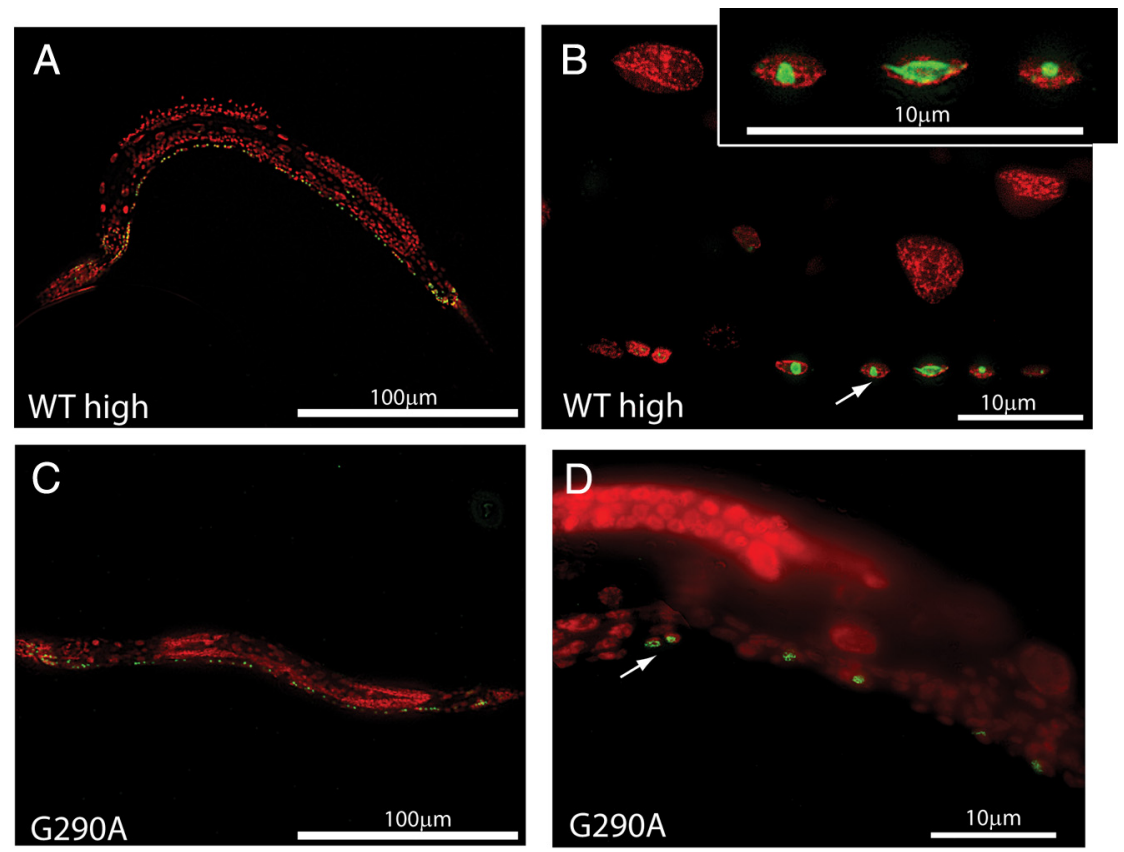

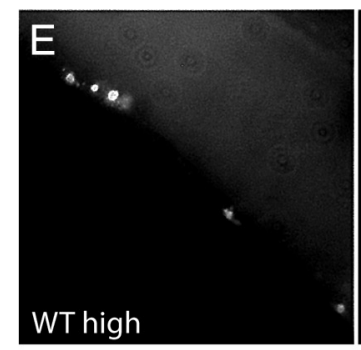

anti-TDP43
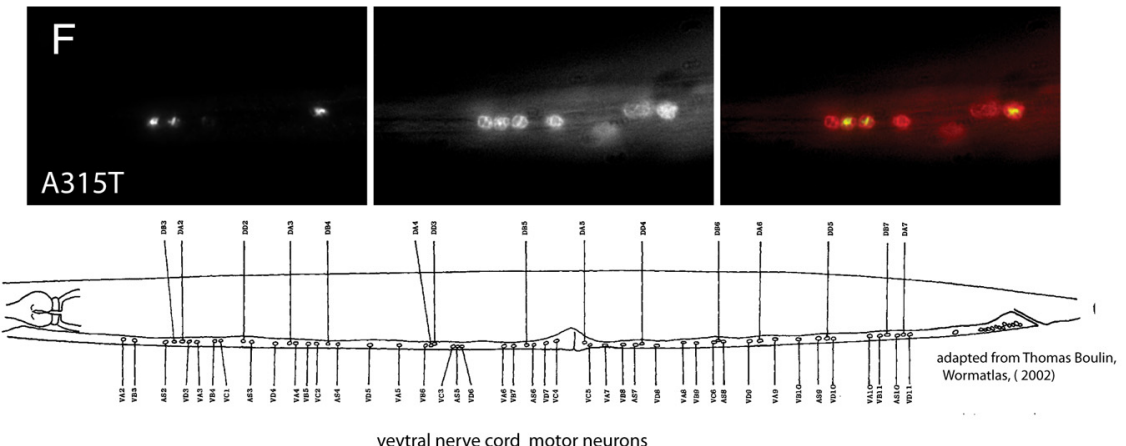

Figure 3. Human TDP-43 accumulates in neuronal nuclear bodies. Freeze-cracked whole worms were immunostained with anti-TDP-43 primary antibody, secondary detection with anti-mouse Alexa 488 (green). Nuclei were labeled with DAPI counterstain (red). Distinct nuclear bodies in motor neurons of the ventral nerve cord (see diagram at bottom) label with TDP-43 antibodies (arrows), reminiscent of neuronal nuclear inclusions. Nuclear localization of TDP-43 appears identical for the wild type $(\boldsymbol{A}, \boldsymbol{B})$ or mutant (G290A) (C, D) TDP-43, and is consistent in all neuronal classes observed (data not shown). $\boldsymbol{E}$ (WT) and $\boldsymbol{F}$ (mutant) (A315T) show channels acquired separately and then merged for clarity. All worms are oriented anterior left, ventral down.

\section{TDP-43-induced neurodegeneration is independent of} caspase activity and apoptotic pathways

Truncated TDP-43 protein species are a feature of authentic TDP-43 pathology seen on immunoblots of postmortem ALS and FTLD-U brain and spinal cord samples (Neumann, 2009). Cleavage by caspase has been implicated in the formation of TDP-43 truncation products. Caspase cleavage is also suggested to play a critical role in TDP-43 pathology (Zhang et al., 2007, 2009). To examine whether caspase cleavage of TDP-43 is necessary for the production of neurotoxic TDP-43, we tested the re- 

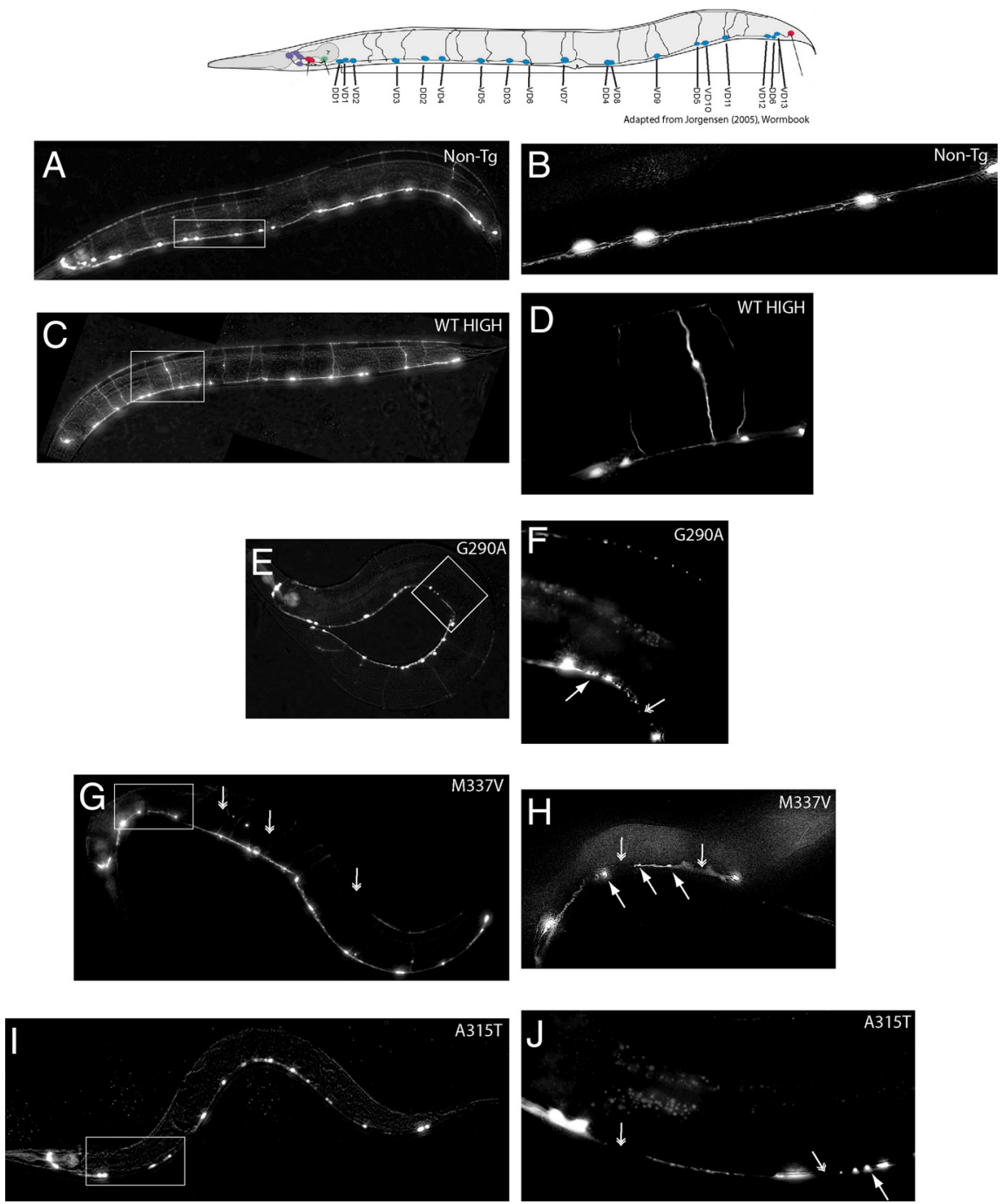

Figure 4. Mutant TDP-43 causes degeneration of GABAergic neurons. Fluorescence microscopy of GFP-labeled VD and DD class GABAergic neurons in living worms. All worms are oriented with anterior end left and ventral side down, as depicted in the schematic of normal GABAergic neurons and processes. The panels on left $(\boldsymbol{A}, \boldsymbol{C}, \boldsymbol{E}, \boldsymbol{G}, \boldsymbol{I})$ show representative whole worms. The panels on right $(\boldsymbol{B}, \boldsymbol{D}, \boldsymbol{F}, \boldsymbol{H}, \boldsymbol{J})$ are high magnification of boxed areas. Non-Tg worms $(\boldsymbol{A}, \boldsymbol{B})$ and worms expressing wild-type TDP-43 $(\boldsymbol{C}, \boldsymbol{D})$ show no obvious defects in the processes and cell bodies of the GABAergic neurons, and no significant neuronal loss (Table 1). In contrast, worms expressing mutant TDP-43 exhibit severe degeneration of neuronal processes (double-headed arrows) and degeneration of cell bodies (arrows). Note complete or near-complete loss of dorsal nerve cord in TDP-43 mutant worms.

quirement for CED-3, the major programmed cell death caspase expressed in C. elegans neurons, and CED-4, a CED-3 activating protein necessary for caspase activity. CED-3 is the only C. elegans caspase known to be expressed in neurons. We crossed ced-3 and ced-4 mutant strains with TDP-43 transgenic lines and assessed motor function. Although both ced-3 and ced-4 mutant strains alone had significantly decreased locomotion compared with the nontransgenic control, they did not exhibit gross abnormalities in locomotion such as coiling or altered waveform. Furthermore, neither loss of ced-3 nor ced-4 altered the visible TDP-43-induced motor abnormalities (Fig. 6A) (data not shown). Worms expressing wild-type TDP-43 had identical radial locomotion in the presence or absence of functional caspase, as did worms expressing mutant TDP-43, indicating the movement defects do not require caspase-dependent truncation of TDP-43. Thus, caspase cleavage of TDP-43 is unlikely to contribute to the neuronal dysfunction caused by TDP-43. Since CED-3 and CED-4 are essential for normal programmed cell death and TDP-43 causes neurodegenerative changes, we also examined whether GABAergic neurons degenerate in ced-3 and ced-4 mutants. To evaluate the role of programmed cell death in TDP-43 neurotoxicity, we crossed mutant TDP-43 M337V and G290A/unc-25::GFP double transgenic lines with either the ced -3 or ced -4 mutants. Loss of ced -3 or ced- 4 fails to alter the timing or course of degeneration of the GABAergic motor neurons (Fig. 6B-E; Table 1, bottom). Furthermore, we examined the TDP-43 protein expressed in ced-3 and ced-4 mutants for alterations in cleavage pattern and see no obvious changes, with fulllength TDP-43 being the predominant species, a relatively abundant $35 \mathrm{kDa}$ truncated product, and low levels of a 25 $\mathrm{kDa}$ product (Fig. 6 F). The relative abundance of cleavage products compared with full-length product is similar to what is observed in the TDP-43 transgenic animals without ced mutations (Figs. 1D, 5). Thus, in C. elegans, we find no evidence for cell death caspase CED-3 participation in the generation of TDP-43 truncated products or participation in neurodegeneration.

\section{Transgenic animals exhibit} ubiquitination and phosphorylation of TDP-43

TDP-43 was initially identified on the basis of its pathological ubiquitination (Arai et al., 2006; Neumann et al., 2006). To investigate whether TDP-43 is ubiquitinated in this model, worm lysates from TDP-43 transgenic animals were subjected to immunoprecipitation with TDP43-specific antibodies. The resulting material contains high-molecular-weight ubiquitinated protein in TDP-43 transgenic lysates but not control lysates from nontransgenic animals consistent with poly ubiquitinated TDP-43 species accumulation (supplemental Fig. S3B, available at www.jneurosci.org as supplemental material). The abundance of ubiquitinated species is in relative proportion to total TDP-43 levels (Fig. 1D).

TDP-43 protein extracted from the CNS of ALS or FTLD-U patients exhibit phosphorylation at several sites, whereas normal control CNS does not have detectable levels of phospho-TDP-43 (Neumann et al., 2006; Hasegawa et al., 2008). Recent work has identified TDP-43 serine residues 409 and 410 as major sites of pathological phosphorylation. These residues are consistently phosphorylated in the TDP-43 inclusions of ALS and FTLD-U but not on nonpathological TDP-43 (Inukai et al., 2008; Neumann et al., 2009).

To address whether TDP-43 is phosphorylated in this model of TDP-43 neurotoxicity, we immunoblotted worm protein fractions analyzed above (Fig. 7A), and probed with antibody pS409/ 410 , which recognizes the C-terminal phospho-serine $409 / 410$ epitope. We observe robust phosphorylation of TDP-43 in strains carrying each of the familial ALS mutations tested, but 
limited phosphorylation of wild-type TDP-43. The TDP-43 WTlow transgenic line demonstrated modest phosphorylation at S409/410 only in the detergent-insoluble fraction, whereas the TDP-43 WT-high transgenic line did not exhibit phosphorylated TDP-43 in any fraction. No modification of the LS fraction was evident in any of the ALS-mutant TDP-43 lines, but all three mutations appear to drive phosphorylation in the TX, SARK, and UREA fractions. As a control, protein samples were dephosphorylated with lambda phosphatase (Hanger et al., 2002) to demonstrate the specificity of the TDP-43 phosphospecific antibody. As expected, all immunoreactivity with the phosphospecific TDP-43 antibody disappeared after sample treatment with lambda phosphatase (supplemental Fig. S6, available at www. jneurosci.org as supplemental material). These results suggest mutant TDP-43 is subject to pathological phosphorylation, whereas normal TDP-43 resists phosphorylation at the $\mathrm{S} 409 / 410$ sites. Mutant TDP-43 lines exhibit greater levels of both neurotoxicity and S409/410 phosphorylation compared with the wild-type TDP-43 lines. Therefore, phosphorylation of TDP-43 may drive toxicity of mutant TDP-43. The low level of phosphoTDP-43 detectable in the WT-low transgenic line, but not in WT-high may explain the mild phenotypic differences between these two strains.

Phosphorylation of TDP-43 at S409/410 is required for neurotoxicity

To test whether phosphorylation of S409/ 410 is important for TDP-43 pathogenesis, we generated TDP-43 transgenes carrying one of two familial ALS mutations (G290A or M337V) as well as double serine-to-alanine mutations at amino acids 409/410, resulting in the ablation of the $\$ 409 / 410$ phosphorylation sites. These phosphorylation-defective transgenes are driven by the pan-neuronal promoter snb-1 (Fig. 1A) and integrated into the $C$. elegans genome. We refer to ALS-mutant transgenes lacking S409/410 phosphorylation sites (alanine substituted for serine) as G290A/AA and M337V/AA, respectively. We examined TDP-43 protein from the G290A/AA and M337V/AA lines and found both G290A/AA and M337V/AA transgenes are not phosphorylated at the pS409/410 sites, but express abundant nonphosphorylated TDP-43 at levels similar to the original G290A and M337V transgenes (supplemental Fig. S5, available at www.jneurosci.org as supplemental material). Examination of locomotion in the G290A/AA and M337V/AA lines revealed a marked amelioration of TDP-43-induced neuronal dysfunction, including reduced paralysis, coiling, and uncoordinated movements, increased responsiveness to stimulation, and nearly normal waveform movements (Fig. 7B; supplemental Movie M337V-AA, available at www.jneurosci.org as supplemental material) (data not shown). When we quantified locomotion data, we observed significant differences between the
Table 1. Measurement of degenerative changes in motor neurons

\begin{tabular}{lllll}
\hline Strain & Neuronal loss & VC axonal gaps & DC axonal gaps & $N$ \\
\hline Non-Tg & $0.2 \pm 0.09$ & $0.3 \pm 0.10$ & $0.1 \pm 0.07$ & 28 \\
WT-high & $0.4 \pm 0.11$ & $0.8 \pm 0.13$ & $0.2 \pm 0.09$ & 20 \\
WT-low & $0.3 \pm 0.10$ & $0.2 \pm 0.09$ & $0.5 \pm 0.14$ & 20 \\
G290A & $3.5 \pm 0.59^{*}$ & $7.3 \pm 1.10^{*}$ & $8.8 \pm 0.52^{*}$ & 22 \\
M337V & $4.2 \pm 0.41^{*}$ & $5.4 \pm 0.51^{*}$ & $4.8 \pm 0.68^{*}$ & 24 \\
A315T & $5.0 \pm 0.57^{*}$ & $6.8 \pm 0.47^{*}$ & $9.7 \pm 0.70^{*}$ & 20 \\
ced-3;M337V & $4.2 \pm 0.43^{*}$ & $6.6 \pm 2.20^{*}$ & $9.6 \pm 0.86^{*}$ & 20 \\
ced-4;G290A & $5.6 \pm 0.70^{*}$ & $7.6 \pm 0.67^{*}$ & $9.5 \pm 0.65^{*}$ & 20
\end{tabular}

Assessment of neurodegenerative changes in GABAergic motor neurons by visual scoring of GFP reporter. Neurons lost reflect the mean number of neurons missing of a total of $19 \mathrm{VD}$ and DD class GABAergic motor neurons \pm SEM. VC gaps are disruptions in the continuity of the ventral nerve cord. DC gaps are disruptions in the continuity of the dorsal nerve cord. $N$ is the number of animals scored. Statistical significance was analyzed using ANOVA (KruskalWallis test); $p$ value reflects the comparison with reporter alone.

${ }^{*} p<0.001$ versus non- $\mathrm{Tg}$

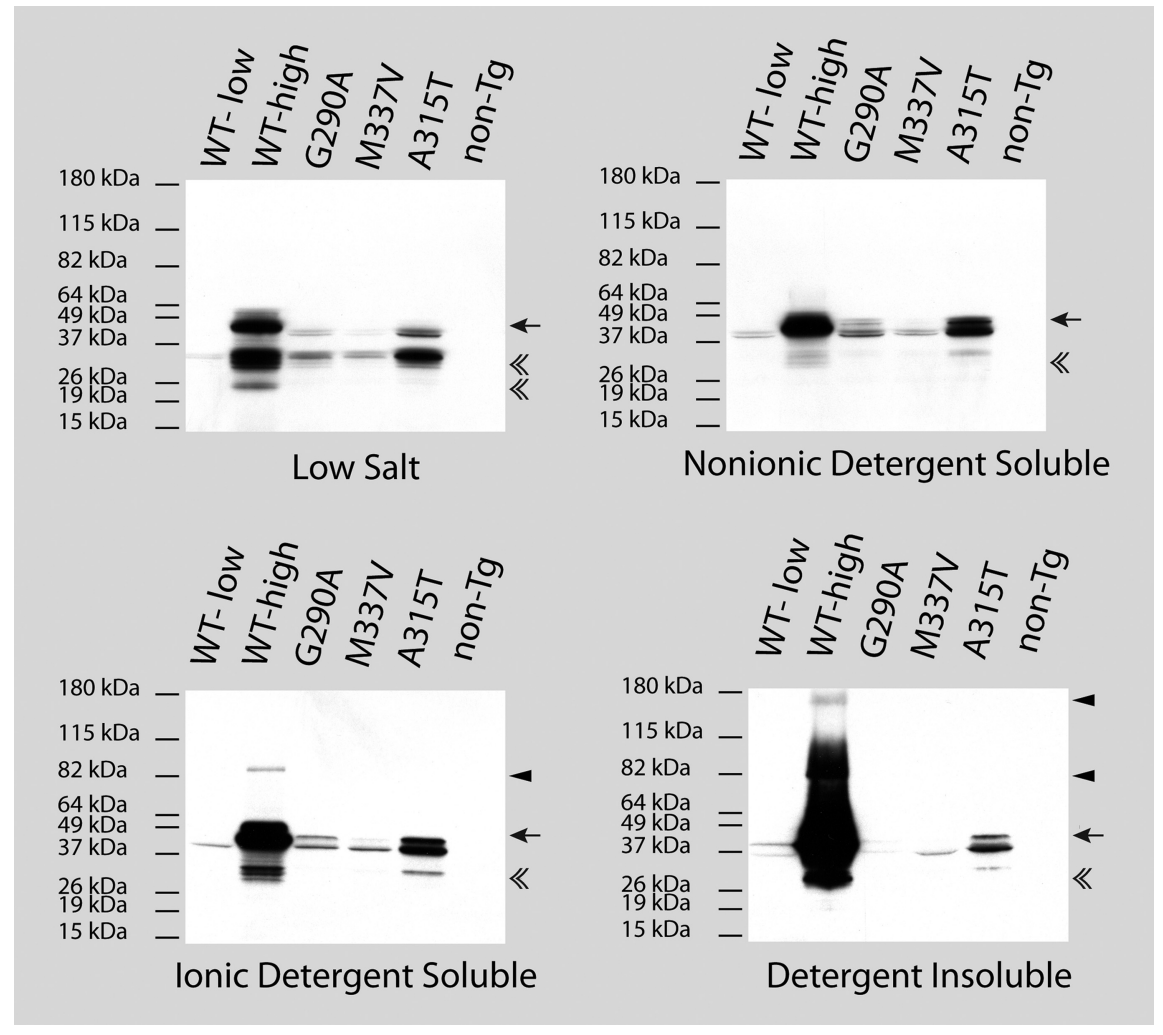

Figure 5. Worm neurons accumulate detergent-insoluble human TDP-43 species. Sequential extraction of insoluble TDP-43 protein was performed from transgenic strains expressing human TDP-43. Equivalent masses of worms were homogenized in low-salt buffer LS, and the supernatant after centrifugation (denoted low-salt) constitutes the fraction of soluble TDP-43. The LS-insoluble material was extracted with TX buffer yielding nonionic detergent-soluble material (nonionic detergent-soluble fraction). The TX-insoluble material was reextracted with ionic detergent containing buffer SARK (ionic detergent-soluble fraction). The SARK-insoluble material was solubilized in $7 \mathrm{~m}$ urea/2 $\mathrm{m}$ thiourea yielding the detergent-insoluble fraction. All fractions were analyzed by immunoblotting using TDP-43-specific antibodies. Strains expressing TDP-43 are as follows: WT-high, WT-low, G290A, M337V, and A315T. The single arrow represents the major full-length isoform of TDP-43 at $\sim 45 \mathrm{kDa}$ in size in all panels. The single arrowheads depict high-molecular-weight species in the ionic detergent-soluble ( $\sim 90 \mathrm{kDa})$ and detergent-insoluble (both $\sim 90$ and $\sim 180 \mathrm{kDa}$ species) fractions likely corresponding to covalently cross-linked dimeric and tetrameric aggregated species. The double arrowheads indicate truncated TDP-43 species present in all fractions $(\sim 35 \mathrm{kDa})$ and in the low-salt fraction (both $\sim 35$ and $\sim 25 \mathrm{kDa}$ species). No TDP-43 is detected in the non-Tg control. phosphorylation-defective mutant lines and the original G290A and M337V lines (Fig. 7C). In fact, ablation of the pS409/410 site reduced the movement abnormalities of worms expressing G290A and M337V ALS-mutant TDP-43 to levels observed with wild-type TDP-43, suggesting phosphorylation at the S409/410 site drives toxicity for these two TDP-43 mutants. 
A
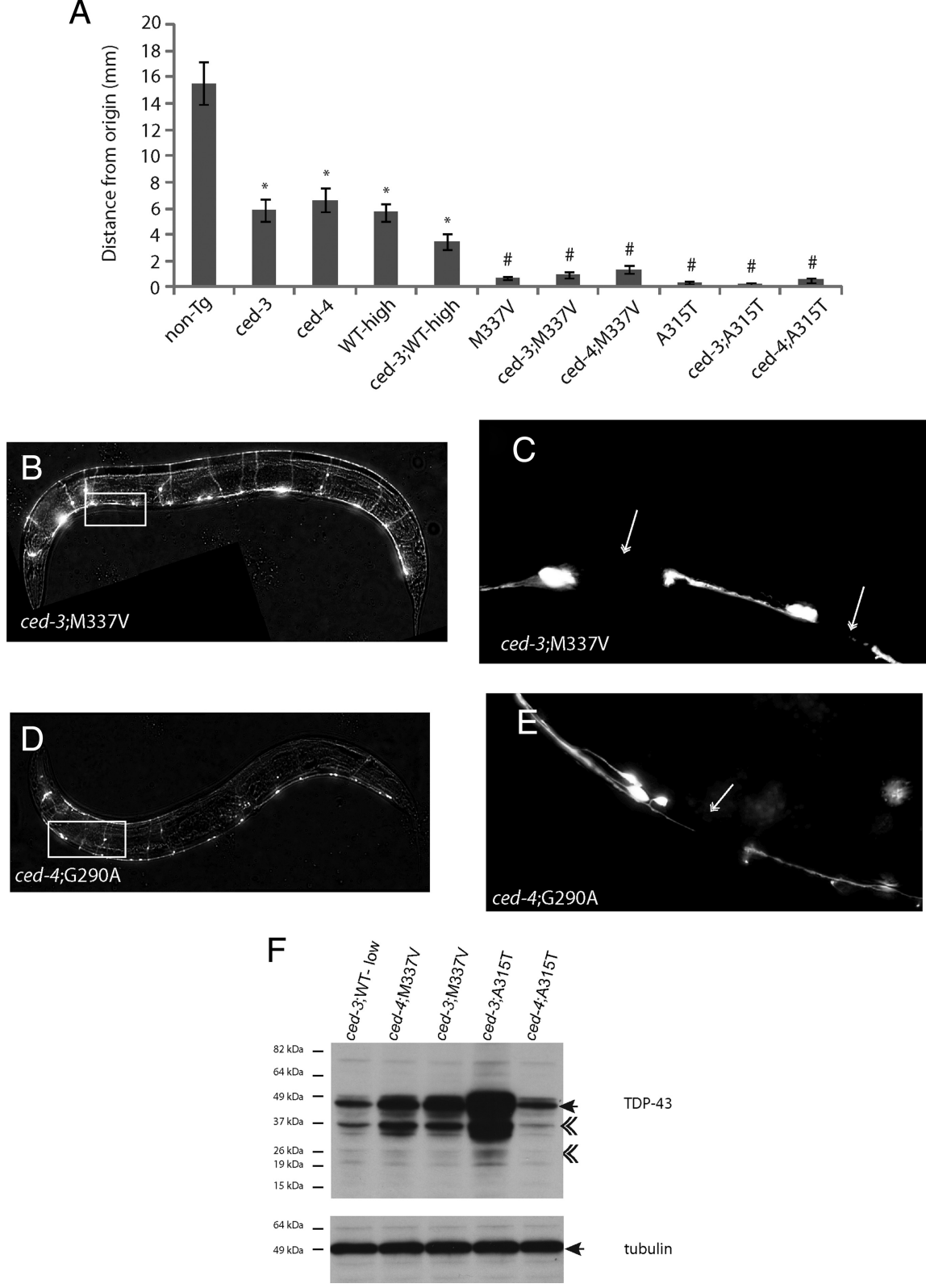

Figure 6. Caspase activity and the apoptotic pathway are not required for TDP-43 neurotoxicity. Loss-of-function mutations in genes necessary for programmed cell death, the caspase ced-3 or its activator ced-4, do not visibly alter the motor phenotype or neurodegeneration caused by ALS-mutant TDP-43. A, Measurement of radial locomotion seen in ced-3, ced-4, and double mutants with TDP-43 transgenes. Measurements are the combination of at least two experiments; $N=55$, non-Tg; 45 , ced-3; 27, ced-4; 60, WT-high; 58, M337V; 48, A315T; 32, ced-3;WT-high; 28, ced-3;M337V; 34, ced-4;M337V; 27, ced-3;A315T; 28, ced-4;A315T. Error bars are SEM. Locomotion of ced-3;WT-high is not significantly different from ced-3 or WT-high despite appearing to have a slight decrease in distance traveled, and locomotion of ced-3;M337V, ced-4;M337V, ced-3;A315T, and ced-4;A315T are not significantly different from M337V and A315T, respectively (one-way ANOVA, Tukey's multiple-comparison test). ${ }^{*} p<0.001$ versus non-Tg; ${ }^{\#} p<0.05$ versus WT-high. $\boldsymbol{B}-\boldsymbol{E}$, Photomicrographs of GFP-labeled GABAergic neurons in worms with loss-of-function mutations in the apoptosis pathway. All worms are oriented anterior left, ventral down. The panels on left $(\boldsymbol{B}, \boldsymbol{D})$ show representative whole worms. The panels on right $(\boldsymbol{C}, \boldsymbol{E})$ are high magnification of boxed areas. Neither loss of ced-3 nor ced-4 alters the GABAergic neurodegenerative changes (double arrows) seen in M337V and G290A in the presence of active CED-3 and CED-4 (Fig. 4E, G, Table 1). $\boldsymbol{F}$, Elimination of CED-3 or CED-4 activity does not alter the abundance of truncated TDP-43 as detected by TDP-43 immunoblotting. The single arrow represents the major full-length isoform of TDP- 43 at $\sim 45 \mathrm{kDa}$ in size. The double arrowheads indicate truncated TDP-43 species present, including $\sim 35$ and $\sim 25 \mathrm{kDa}$ species at the predicted size of TDP-43 caspase cleavage products. Tubulin is used as a loading control.

To control for potential effects of TDP-43 S409/410 substitutions, we generated several transgenic strains carrying either chromosomally integrated or extrachromosomal transgenes expressing human TDP-43 with S409/410A substitutions in the absence of ALS causing mutations, and observed no change in locomotion compared with wild-type TDP-43 (supplemental Fig. S7, available at www.jneurosci.org as supplemental material). Thus, phosphorylation at $S 409 / 410$ is not necessary for toxicity of wild-type TDP-43, and substitution of serines 409 and 410 with alanines does not on its own diminish TDP-43 toxicity. We also tested transgenic lines of non-ALSmutant TDP-43 with substitutions of serine 409 with aspartic acid (S409D) or serines 409 and 410 with aspartic acid or glutamic acid (S409/410D and S409/410E, respectively) to mimic the charge difference created by phosphorylation at those sites. However, when these substitutions in TDP-43 were expressed using either extrachromasomal or integrated transgenes, we did not observe any change in the locomotion defects of the transgenic animals (supplemental Fig. S7, available at www. jneurosci.org as supplemental material).

To test whether phosphorylated TDP-43 has altered subcellular localization compared with nonphosphorylated TDP-43, we examined pS409/410 TDP-43 immunostaining in ALS-mutant TDP-43 transgenic lines. We observed that pS409/410 antibody staining shares a similar nuclear staining pattern with pan-TDP-43 (Fig. $7 D, E)$.

\section{Discussion}

Expression of mutated human TDP-43encoding CDNAs in worm neurons results in a disease model recapitulating key features of human TDP-43 proteinopathy disorders including motor and behavioral abnormalities, reduced life span, accumulation of detergent-insoluble, ubiquitinated, and phosphorylated abnormal TDP-43 protein species, and neurodegenerative changes (for a summary of phenotypes, see supplemental Table S2, available at www. jneurosci.org as supplemental material). Expression of neither low nor high levels of normal human TDP-43 in transgenic C. elegans results in significant neurodegeneration, but does lead to moderate and progressive motor dysfunction. However, familial ALS-mutant versions of TDP-43 (G290A, M337V, or A315T) expressed at similar levels cause a severe and progressive motor phenotype indicative of significant motor neuron dysfunction (Fig. 1) demonstrating the neurotoxic effect of the TDP-43 mutations. In ALS and FTLD-U, disease phenotypes worsen over time. Likewise, in this model expression of TDP-43 leads to progressive paralysis (Fig. 2) and reduced life span. Interestingly, in worms the correlation between average life span and severity of motor dysfunction is limited (Lakowski and 
Hekimi, 1998; Ailion et al., 1999; Muñoz and Riddle, 2003). The disconnect between life span and motor function may be attributable to the opposing effects of paralysis-induced caloric restriction and rapid aging because of motor dysfunction. The WT-low-expressing line has a shorter life span and slightly worse locomotion than the WT-high, despite lower levels of TDP-43. However, the WT-low line has low levels of detectable S409/410 phosphorylation present only in its detergentinsoluble fraction (Fig. 7A), whereas WT-high does not have S409/410 phosphorylation in any fraction. The relatively worse phenotype of WT-low compared with WT-high is consistent with the hypothesis linking S409/410 phosphorylation to TDP-43 toxicity.

TDP-43 is predicted to contain both nuclear localization and nuclear export signals (Ayala et al., 2008; Winton et al., 2008), indicative of a cellular function that involves shuttling between the nucleus and cytoplasm. Although TDP-43 is normally found in the nucleus, it can relocate to the cytoplasm in response to neuronal injury (Moisse et al., 2009). Abnormal TDP-43 forms pathologic inclusions in both compartments with varying frequency in different TDP-43 proteinopathy disorders. For instance, cytoplasmic neuronal and glial inclusions are common in ALS, but vary widely in subtypes of FTLD-U. Nuclear inclusions of TDP-43 are common in some subtypes of FTLD-U, such as those caused by progranulin mutations, but rare in others (Forman et al., 2007). Nuclear neuronal inclusions containing TDP-43 also predominate in IBMPFD (inclusion body myopathy associated with Paget's disease of bone and frontotemporal dementia). Likewise, in this model of TDP-43 proteinopathy, aggregated TDP-43 localizes predominantly to the nucleus of neurons for both normal and mutated TDP-43 transgenic lines (Fig. 3). Although some studies demonstrate cytoplasmic localization of TDP-43 in response to ALS causing mutations (Barmada et al., 2010), in others nuclear localization of aggregates predominates and drives neurodegeneration (Wegorzewska et al., 2009). The lack of correlation between TDP-43 cellular localization and neurotoxicity may indicate that localization alone is not responsible for TDP-43-driven neurodegeneration.

Loss of motor neurons is a key finding in postmortem analysis of the nervous systems of ALS patients. Given the prominent motor phenotype in TDP- 43 transgenic animals, we examined the effects of wild-type and familial ALS-mutant forms of TDP-43 on neurons in living animals. Interestingly, worms expressing wild-type TDP-43 did not exhibit GABAergic neurodegeneration, despite obvious motor deficits. However, we observe progressive and severe degeneration of the VD and DD classes of

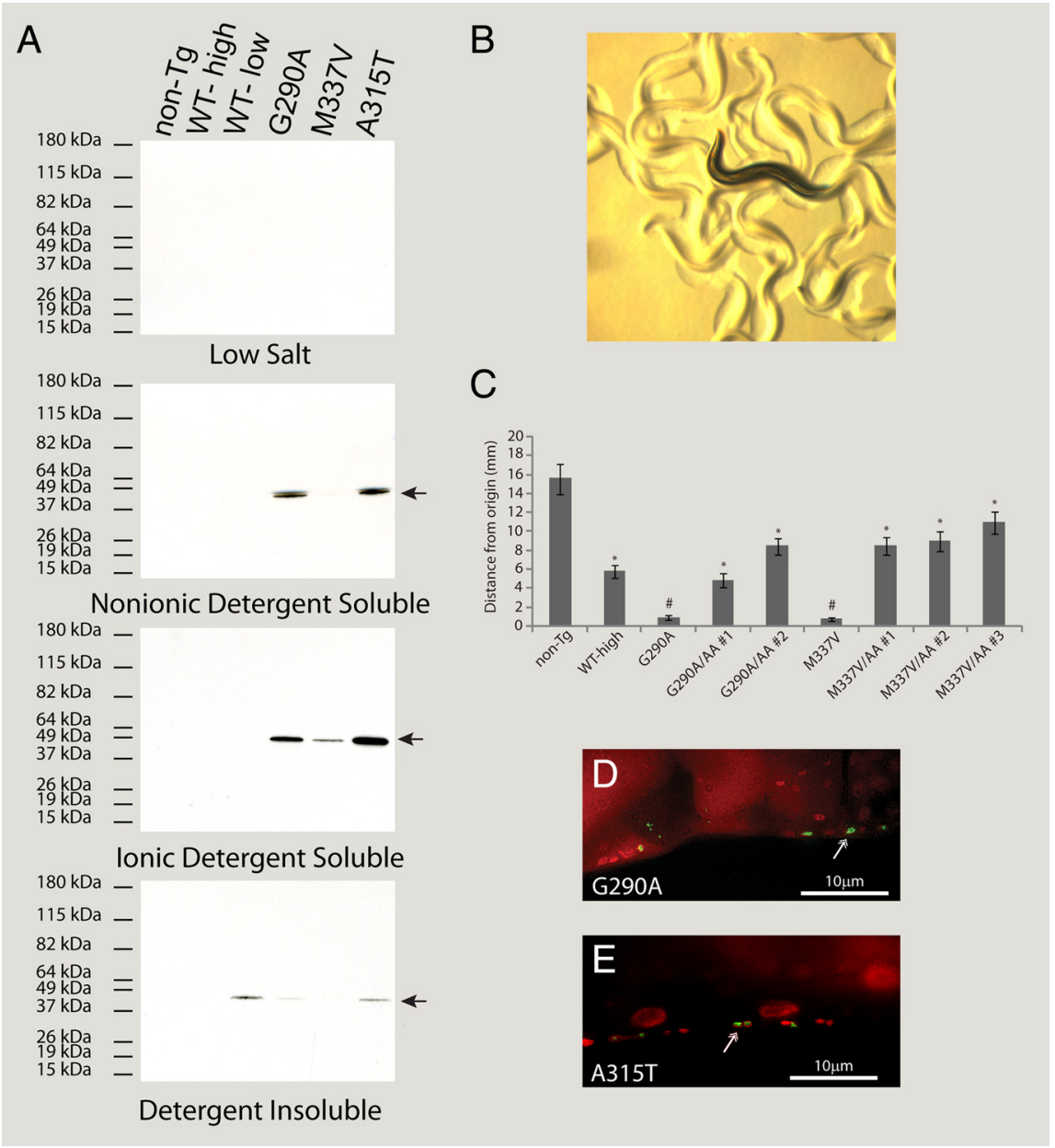

Figure 7. Phosphorylation at $\$ 409 / 410$ is required for ALS-mutant TDP-43 neurotoxicity. A, TDP-43 transgenic worms were sequentially extracted, as shown in Figure 5, and probed with a TDP-43 antibody specifically recognizing TDP-43 phosphorylated at 5409/410. The WT-high and WT-low fractions have little or no detectible phosphorylated TDP-43 present in any of the soluble or insoluble fractions. In contrast, the mutant TDP-43 strains all contain phosphorylated bands in the ionic detergent-soluble fraction, s indicated by the black arrow. B, Phosphorylation-null mutants G290A/AA and M337V/AA movement is restored to that observed in werms expsing wild-type TDP-43, with reduced paralysis, improved locomotion, and decreased coiling compared with G290A (is M337V/AA. C, SS409/410AA mutations restore G290A and M337V locomotion to the levels observed in s expressing wild-type TDP-43. N = 55, non-Tg; 60, WT-high;32, G290A;58, M337V;25, G290A/AA\#1;35, G290A/AA\#2; 47, were labeled with DAPI counterstain (red). The double arrow indicates TDP-43 phosphorylated at $\$ 409 / 410$ with similar subcellular localization to non-phospho-TDP-43 (Fig. 4). All worms are oriented anterior left, ventral down.

GABAergic motor neurons in animals expressing familial ALSmutant forms of TDP-43 (Fig. 4). We also observed degeneration of ADE and CEP but not PDE classes of dopaminergic neurons in mutant but not wild-type TDP-43 lines (data not shown). Neurodegeneration in mutant but not wild-type TDP-43-expressing animals likely underlies the differences in severity of motor dysfunction in mutant versus wild-type TDP-43-expressing animals, and may underlie additional sensory or behavioral problems yet to be explored in this model. It is likely that mutant TDP-43 neurodegeneration is limited to specific subsets of neurons in $C$. elegans, since worms expressing mutant TDP-43 are viable and have grossly normal development and adult morphology. Whether TDP-43 destroys any additional populations of neurons and, if so, analyzing what features they share with GABAergic and 
dopaminergic neurons will be important future work. Human TDP-43 proteinopathies feature degeneration of specific subtypes of neurons, and worms provide a tractable system to explore the specificity of TDP-43 neurodegeneration.

Pathological lesions containing detergent-insoluble, phosphorylated, and aggregated TDP-43 are a key feature of TDP-43 proteinopathies like FTLD-U and ALS and can be found in both the nucleus and cytoplasm of neurons (for review, see Gendron et al., 2010; Geser et al., 2010). The vast majority of TDP-43 proteinopathy cases exhibit abnormal TDP-43 aggregates in the absence of TDP-43 mutations. Similarly, in this model we see TDP-43 detergent-insoluble aggregates independent of ALS causing TDP-43 mutations (Fig. 5). However, the severity of neuronal dysfunction or degeneration does not correlate with protein aggregation. This observation suggests aggregation of TDP-43 does not drive neurotoxicity, because although mutant TDP-43 is decidedly more toxic than wild-type TDP-43, we observe considerably more aggregated wild-type TDP-43 protein than mutant TDP-43 protein. Thus, we conclude that mutant and wild-type TDP-43 cause neuronal dysfunction by distinct mechanisms or aggregation does not play a causal role in neurotoxicity, but rather may be a cellular consequence of TDP-43 dysfunction.

Caspase cleavage of TDP-43 has been suggested to be important in the pathogenic mechanism of ALS and FTLD-U (Zhang et al., 2009), and caspases are known to mediate apoptotic cell death. We observe truncated species in all TDP-43 transgenic lines approximately in proportion to the level of full-length TDP-43 protein expressed (Fig. $1 D$ ). Thus, we explored the requirements for ced-3 and ced-4 in TDP-43-mediated motor dysfunction and neurotoxicity. In C. elegans, ced-3 is a caspase gene essential for apoptosis, whereas ced-4 is a caspase activator gene required for normal apoptosis. Loss of ced-3 or ced-4 function does not prevent wild-type or mutant TDP-43-induced movement defects or neurodegeneration (Fig. 6) demonstrating that caspase-dependent cleavage and activation of conventional apoptosis pathways are not required in the pathology and neurotoxicity of TDP-43. Furthermore, truncated TDP-43 products persist in the absence of the CED-3 caspase (Fig. 6 F) (Nishimoto et al., 2010). Likewise, we see little relationship between the level of truncated species and the degree of neurodegeneration, since TDP-43 WT-high has the highest level of truncated species and TDP-43 WT-low has the lowest level of truncated species (Fig. 5), yet neither has significant neurodegenerative changes. These findings are consistent with observations in cultured mammalian cells, where TDP-43 fragments persist in the absence of caspase function (Nishimoto et al., 2010). It is possible other mechanisms of cell death such as necrosis or cellular atrophy are contributing to TDP-43-driven neurodegeneration (Bodansky et al., 2010). Future work will address more precisely what mechanism is involved in neuronal death in this TDP-43 model.

The theme of aberrant phosphorylation of misfolded or aggregated proteins has been noted in the pathological characterization of several neurodegenerative diseases in addition to ALS and FTLD-U. For instance, hyperphosphorylation of microtubule binding protein tau is a diagnostic hallmark of Alzheimer's disease (for review, see Buée et al., 2000), whereas hyperphosphorylation of $\alpha$-synuclein occurs in Parkinson's disease (for review, see Thomas and Beal, 2007; Kahle, 2008). Whether phosphorylation is a cause or consequence of protein aggregation and neurotoxicity remains a debated point for both disorders. Interestingly, we see marked enrichment of pS409/410 species in ALS-mutant TDP-43-expressing lines (Fig. 7A, nonionic detergent and ionic detergent fractions), correlating TDP-43 phosphorylation with the severity of motor dysfunction and neurodegeneration.

In ALS-mutant TDP-43 lines, phosphorylated TDP-43 is only observed in detergent-soluble and -insoluble protein fractions, indicating the toxic phosphorylated species may be misfolded. This may also hold true for the differences observed in the WTlow- versus WT-high-expressing lines. The more toxic WT-low line has low-level, but detectable phosphorylated TDP-43, whereas WT-high has none (Fig. 7A). The abundance of phosphorylation of ALS mutated TDP-43 compared with low or undetectable phospho-TDP-43 in wild-type lines highlights potentially different mechanisms for toxicity of wild-type and ALSmutant TDP-43. Furthermore, we demonstrate that ALS-mutant TDP-43 is no more toxic than wild-type TDP-43 when phosphorylation of the S409/410 site is prevented (Fig. 7); however, this same removal of phosphorylation has no effect on the toxicity of otherwise wild-type TDP-43 (supplemental Fig. S7B, available at www.jneurosci.org as supplemental material). Likewise, pseudophosphorylation on its own does not cause any dramatic increase in TDP-43 toxicity (supplemental Fig. S7, available at www.jneurosci.org as supplemental material), suggesting either that phosphorylated TDP-43 is only toxic in combination with ALS causing mutations, or that pseudophosphorylation at positions 409/410 does not accurately recapitulate the structural changes of authentic phosphorylation of TDP-43 at S409/410.

Previous pathological analysis of postmortem ALS and FTLD-U CNS samples clearly demonstrates S409/410 phosphorylation in TDP-43 lesions, but not normal CNS tissue (Inukai et al., 2008; Neumann et al., 2009). Together, previously published pathological and functional findings (Inukai et al., 2008; Neumann et al., 2009) and our data support a critical role for TDP-43 phosphorylation in the mechanism of neurodegeneration in familial ALS disease.

TDP-43 has been the focus of intensive study since its identification as the pathological protein in ALS and FTLD-U ubiquitinated inclusions (Arai et al., 2006; Neumann et al., 2006). Efforts to understand the mechanism of disease have led to the development of model systems exhibiting some features of TDP-43 pathology. The first mammalian model was recently described demonstrating viral-mediated expression of wild-type human TDP-43 in the substantia nigra of rats. These animals show loss of dopaminergic neurons and display behavioral phenotypes. They also mimic some features of TDP-43 pathology including cytoplasmic localization and ubiquitination (Tatom et al., 2009). A mouse model expressing wild-type human TDP-43 in most CNS neurons displayed dose-dependent degeneration of motor neurons, spastic quadriplegia, accumulation of truncated, detergent-insoluble TDP-43 products, and shortened life span (Wils et al., 2010). Expression of ALS-mutant TDP-43 (M337V) in rats led to widespread neurodegeneration, muscle atrophy, paralysis, and shortened life span, phenotypes that were not observed with expression of wild-type human TDP-43 (Zhou et al., 2010). Another recent study describes neuronal expression of ALS-mutant TDP-43 (A315T) in mice, resulting in selective neurodegeneration, progressive gait abnormalities, and shortened life span (Wegorzewska et al., 2009). Our observations that high levels of detergent-insoluble TDP-43 can occur without significant neurodegeneration (summarized in supplemental Table S2, available at www.jneurosci.org as supplemental material) are consistent with the mouse and rat models of mutant TDP-43 neurotoxicity, where TDP-43 exhibited little or no aggregation or inclusion formation (Wegorzewska et al., 2009; Zhou et al., 2010). 
This indicates TDP-43-driven neurodegeneration can occur in the absence of protein aggregation.

C. elegans is a simple system allowing us to model the cellular and molecular changes in neurodegeneration, although it cannot accurately model more complex pathological features associated with mammalian nervous system dynamics. For instance, the selective and variable neuronal vulnerability in FTLD-U (Seeley et al., 2006) or the focal onset, progression, and spreading of ALS (for review, see Ravits and La Spada, 2009) require a model with the complexity of the mammalian CNS architecture. These questions can best be addressed in the study of vertebrate animal models of neurodegeneration or in study of patients themselves. However, this C. elegans model of TDP-43 proteinopathies is highly relevant to understanding TDP-43-related disease mechanisms because of its potential for dissection of the determinants of TDP-43 neurotoxicity at the cellular and molecular levels. For instance, we demonstrate the reversal of the motor dysfunction phenotype of ALS-mutant TDP-43 but not wild-type TDP-43 with ablation of the serine $409 / 410$ phosphorylation sites and the subsequent loss of phospho-TDP-43 protein. Although this model lacks the cytoplasmic mislocalization of TDP-43 commonly seen in ALS, we do demonstrate specific and robust nuclear aggregation of TDP-43 without any detectable cytoplasmic TDP-43 (Fig. 3). This nuclear aggregation of TDP-43 is not typical of ALS, but is commonly seen in some FTLD-U cases. Thus, we present this as a generic model of TDP-43 proteinopathy rather than a specific model of a single human disease.

Use of $C$. elegans as a model system permits unbiased approaches to genetically manipulate a model of human neurodegenerative diseases (Kraemer et al., 2006; Kuwahara et al., 2008; Guthrie et al., 2009). This TDP-43 proteinopathy model is likewise tractable for genome-wide forward and reverse genetic screens, which will allow identification of novel modifiers of TDP-43 activity. Furthermore, this model will facilitate exploration of the mechanism of TDP-43 motor neurotoxicity, including dissection of which molecular features of TDP-43 pathology cause motor neuron dysfunction, and which are secondary effects or markers of motor neuron degeneration. In addition, the worm model has potential as a system to rapidly screen for pharmacological modifiers of TDP-43 pathology, potentially guiding development of therapeutic interventions. For example, identification of compounds or mutations preventing phosphorylation of TDP-43 at S409/410 may be important neuroprotective strategies for TDP-43 proteinopathy disorders.

\section{References}

Ailion M, Inoue T, Weaver CI, Holdcraft RW, Thomas JH (1999) Neurosecretory control of aging in Caenorhabditis elegans. Proc Natl Acad Sci U S A 96:7394-7397.

Arai T, Hasegawa M, Akiyama H, Ikeda K, Nonaka T, Mori H, Mann D, Tsuchiya K, Yoshida M, Hashizume Y, Oda T (2006) TDP-43 is a component of ubiquitin-positive tau-negative inclusions in frontotemporal lobar degeneration and amyotrophic lateral sclerosis. Biochem Biophys Res Commun 351:602-611.

Ayala YM, Misteli T, Baralle FE (2008) TDP-43 regulates retinoblastoma protein phosphorylation through the repression of cyclin-dependent kinase 6 expression. Proc Natl Acad Sci U S A 105:3785-3789.

Barmada SJ, Skibinski G, Korb E, Rao EJ, Wu JY, Finkbeiner S (2010) Cytoplasmic mislocalization of TDP-43 is toxic to neurons and enhanced by a mutation associated with familial amyotrophic lateral sclerosis. J Neurosci 30:639-649.

Benajiba L, Le Ber I, Camuzat A, Lacoste M, Thomas-Anterion C, Couratier P, Legallic S, Salachas F, Hannequin D, Decousus M, Lacomblez L, Guedj E, Golfier V, Camu W, Dubois B, Campion D, Meininger V, Brice A (2009) TARDBP mutations in motoneuron disease with frontotemporal lobar degeneration. Ann Neurol 65:470-473.
Bodansky A, Kim JM, Tempest L, Velagapudi A, Libby R, Ravits J (2010) TDP-43 and ubiquitinated cytoplasmic aggregates in sporadic ALS are low frequency and widely distributed in the lower motor neuron columns independent of disease spread. Amyotroph Lateral Scler 11:321-327.

Brenner S (1974) The genetics of Caenorhabditis elegans. Genetics 77:71-94. Buée L, Bussière T, Buée-Scherrer V, Delacourte A, Hof PR (2000) Tau protein isoforms, phosphorylation and role in neurodegenerative disorders. Brain Res Brain Res Rev 33:95-130.

Buratti E, Baralle FE (2008) Multiple roles of TDP-43 in gene expression, splicing regulation, and human disease. Front Biosci 13:867-878.

Buratti E, Dörk T, Zuccato E, Pagani F, Romano M, Baralle FE (2001) Nuclear factor TDP-43 and SR proteins promote in vitro and in vivo CFTR exon 9 skipping. EMBO J 20:1774-1784.

Buratti E, Brindisi A, Giombi M, Tisminetzky S, Ayala YM, Baralle FE (2005) TDP-43 binds heterogeneous nuclear ribonucleoprotein A/B through its C-terminal tail: an important region for the inhibition of cystic fibrosis transmembrane conductance regulator exon 9 splicing. J Biol Chem 280:37572-37584.

Cinar H, Keles S, Jin Y (2005) Expression profiling of GABAergic motor neurons in Caenorhabditis elegans. Curr Biol 15:340-346.

Collins JJ, Huang C, Hughes S, Kornfeld K (2008) The measurement and analysis of age-related changes in Caenorhabditis elegans. WormBook $1-21$.

Crittenden SL, Kimble J (1999) Confocal methods for Caenorhabditis elegans. Methods Mol Biol 122:141-151.

Forman MS, Trojanowski JQ, Lee VM (2007) TDP-43: a novel neurodegenerative proteinopathy. Curr Opin Neurobiol 17:548-555.

Gendron TF, Josephs KA, Petrucelli L (2010) TDP-43: mechanisms of neurodegeneration. Neuropathol Appl Neurobiol. 36:97-112.

Geser F, Lee VM, Trojanowski JQ (2010) Amyotrophic lateral sclerosis and frontotemporal lobar degeneration: a spectrum of TDP-43 proteinopathies. Neuropathology 30:103-112.

Gitcho MA, Baloh RH, Chakraverty S, Mayo K, Norton JB, Levitch D, Hatanpaa KJ, White CL 3rd, Bigio EH, Caselli R, Baker M, Al-Lozi MT, Morris JC, Pestronk A, Rademakers R, Goate AM, Cairns NJ (2008) TDP-43 A315T mutation in familial motor neuron disease. Ann Neurol 63:535-538.

Guthrie CR, Schellenberg GD, Kraemer BC (2009) SUT-2 potentiates tauinduced neurotoxicity in Caenorhabditis elegans. Hum Mol Genet 18:1825-1838.

Hanger DP, Gibb GM, de Silva R, Boutajangout A, Brion JP, Revesz T, Lees AJ, Anderton BH (2002) The complex relationship between soluble and insoluble tau in tauopathies revealed by efficient dephosphorylation and specific antibodies. FEBS Lett 531:538-542.

Hasegawa M, Arai T, Nonaka T, Kametani F, Yoshida M, Hashizume Y, Beach TG, Buratti E, Baralle F, Morita M, Nakano I, Oda T, Tsuchiya K, Akiyama H (2008) Phosphorylated TDP-43 in frontotemporal lobar degeneration and amyotrophic lateral sclerosis. Ann Neurol 64:60-70.

Huang C, Xiong C, Kornfeld K (2004) Measurements of age-related changes of physiological processes that predict lifespan of Caenorhabditis elegans. Proc Natl Acad Sci U S A 101:8084-8089.

Igaz LM, Kwong LK, Xu Y, Truax AC, Uryu K, Neumann M, Clark CM, Elman LB, Miller BL, Grossman M, McCluskey LF, Trojanowski JQ, Lee VM (2008) Enrichment of C-terminal fragments in TAR DNA-binding protein- 43 cytoplasmic inclusions in brain but not in spinal cord of frontotemporal lobar degeneration and amyotrophic lateral sclerosis. Am J Pathol 173:182-194.

Inukai Y, Nonaka T, Arai T, Yoshida M, Hashizume Y, Beach TG, Buratti E, Baralle FE, Akiyama H, Hisanaga S, Hasegawa M (2008) Abnormal phosphorylation of Ser409/410 of TDP-43 in FTLD-U and ALS. FEBS Lett 582:2899-2904.

Jorgensen EM (2005) GABA. WormBook 1-13.

Kabashi E, Valdmanis PN, Dion P, Spiegelman D, McConkey BJ, Vande Velde C, Bouchard JP, Lacomblez L, Pochigaeva K, Salachas F, Pradat PF, Camu W, Meininger V, Dupre N, Rouleau GA (2008) TARDBP mutations in individuals with sporadic and familial amyotrophic lateral sclerosis. Nat Genet 40:572-574.

Kahle PJ (2008) alpha-Synucleinopathy models and human neuropathology: similarities and differences. Acta Neuropathol 115:87-95.

Kertesz A (2009) Clinical features and diagnosis of frontotemporal dementia. Front Neurol Neurosci 24:140-148. 
Kraemer BC, Schellenberg GD (2007) SUT-1 enables tau-induced neurotoxicity in C. elegans. Hum Mol Genet 16:1959-1971.

Kraemer BC, Zhang B, Leverenz JB, Thomas JH, Trojanowski JQ, Schellenberg GD (2003) Neurodegeneration and defective neurotransmission in a Caenorhabditis elegans model of tauopathy. Proc Natl Acad Sci U S A 100:9980-9985.

Kraemer BC, Burgess JK, Chen JH, Thomas JH, Schellenberg GD (2006) Molecular pathways that influence human tau-induced pathology in Caenorhabditis elegans. Hum Mol Genet 15:1483-1496.

Kuwahara T, Koyama A, Koyama S, Yoshina S, Ren CH, Kato T, Mitani S, Iwatsubo T (2008) A systematic RNAi screen reveals involvement of endocytic pathway in neuronal dysfunction in alpha-synuclein transgenic $C$. elegans. Hum Mol Genet 17:2997-3009.

Lakowski B, Hekimi S (1998) The genetics of caloric restriction in Caenorhabditis elegans. Proc Natl Acad Sci U S A 95:13091-13096.

Lakso M, Vartiainen S, Moilanen AM, Sirviö J, Thomas JH, Nass R, Blakely RD, Wong G (2003) Dopaminergic neuronal loss and motor deficits in Caenorhabditis elegans overexpressing human alpha-synuclein. J Neurochem 86:165-172.

Liachko N, Davidowitz R, Lee SS (2009) Combined informatic and expression screen identifies the novel DAF-16 target HLH-13. Dev Biol 327:97105.

Moisse K, Volkening K, Leystra-Lantz C, Welch I, Hill T, Strong MJ (2009) Divergent patterns of cytosolic TDP-43 and neuronal progranulin expression following axotomy: implications for TDP-43 in the physiological response to neuronal injury. Brain Res 1249:202-211.

Muñoz MJ, Riddle DL (2003) Positive selection of Caenorhabditis elegans mutants with increased stress resistance and longevity. Genetics 163: 171-180.

Neumann M (2009) Molecular neuropathology of TDP-43 proteinopathies. Int J Mol Sci 10:232-246.

Neumann M, Sampathu DM, Kwong LK, Truax AC, Micsenyi MC, Chou TT, Bruce J, Schuck T, Grossman M, Clark CM, McCluskey LF, Miller BL, Masliah E, Mackenzie IR, Feldman H, Feiden W, Kretzschmar HA, Trojanowski JQ, Lee VM (2006) Ubiquitinated TDP-43 in frontotemporal lobar degeneration and amyotrophic lateral sclerosis. Science 314:130-133.

Neumann M, Kwong LK, Lee EB, Kremmer E, Flatley A, Xu Y, Forman MS, Troost D, Kretzschmar HA, Trojanowski JQ, Lee VM (2009) Phosphorylation of S409/410 of TDP-43 is a consistent feature in all sporadic and familial forms of TDP-43 proteinopathies. Acta Neuropathol 117:137-149.

Nishimoto Y, Ito D, Yagi T, Nihei Y, Tsunoda Y, Suzuki N (2010) Characterization of alternative isoforms and inclusion body of the TAR DNAbinding protein-43. J Biol Chem 285:608-619.

Ou SH, Wu F, Harrich D, García-Martínez LF, Gaynor RB (1995) Cloning and characterization of a novel cellular protein, TDP-43, that binds to human immunodeficiency virus type 1 TAR DNA sequence motifs. J Virol 69:3584-3596.

Pesiridis GS, Lee VM, Trojanowski JQ (2009) Mutations in TDP-43 link glycine-rich domain functions to amyotrophic lateral sclerosis. Hum Mol Genet 18:R156-R162.

Ravits JM, La Spada AR (2009) ALS motor phenotype heterogeneity, focality, and spread: deconstructing motor neuron degeneration. Neurology 73:805-811.

Robatzek M, Thomas JH (2000) Calcium/calmodulin-dependent protein kinase II regulates Caenorhabditis elegans locomotion in concert with a $\mathrm{G}_{\mathrm{o}} / \mathrm{G}_{\mathrm{q}}$ signaling network. Genetics 156:1069-1082.

Rutherford NJ, Zhang YJ, Baker M, Gass JM, Finch NA, Xu YF, Stewart H, Kelley BJ, Kuntz K, Crook RJ, Sreedharan J, Vance C, Sorenson E, Lippa C, Bigio EH, Geschwind DH, Knopman DS, Mitsumoto H, Petersen RC, Cashman NR, et al. (2008) Novel mutations in TARDBP (TDP-43) in patients with familial amyotrophic lateral sclerosis. PLoS Genet 4:e1000193.

Seeley WW, Carlin DA, Allman JM, Macedo MN, Bush C, Miller BL, Dearmond SJ (2006) Early frontotemporal dementia targets neurons unique to apes and humans. Ann Neurol 60:660-667.

Sreedharan J, Blair IP, Tripathi VB, Hu X, Vance C, Rogelj B, Ackerley S, Durnall JC, Williams KL, Buratti E, Baralle F, de Belleroche J, Mitchell JD, Leigh PN, Al-Chalabi A, Miller CC, Nicholson G, Shaw CE (2008) TDP-43 mutations in familial and sporadic amyotrophic lateral sclerosis. Science 319:1668-1672.

Tatom JB, Wang DB, Dayton RD, Skalli O, Hutton ML, Dickson DW, Klein RL (2009) Mimicking aspects of frontotemporal lobar degeneration and Lou Gehrig's disease in rats via TDP-43 overexpression. Mol Ther 17:607-613.

Thomas B, Beal MF (2007) Parkinson's disease. Hum Mol Genet 16: R183-R194.

Van Deerlin VM, Leverenz JB, Bekris LM, Bird TD, Yuan W, Elman LB, Clay D, Wood EM, Chen-Plotkin AS, Martinez-Lage M, Steinbart E, McCluskey L, Grossman M, Neumann M, Wu IL, Yang WS, Kalb R, Galasko DR, Montine TJ, Trojanowski JQ, et al. (2008) TARDBP mutations in amyotrophic lateral sclerosis with TDP-43 neuropathology: a genetic and histopathological analysis. Lancet Neurol 7:409-416.

Wang IF, Wu LS, Shen CK (2008) TDP-43: an emerging new player in neurodegenerative diseases. Trends Mol Med 14:479-485.

Wegorzewska I, Bell S, Cairns NJ, Miller TM, Baloh RH (2009) TDP-43 mutant transgenic mice develop features of ALS and frontotemporal lobar degeneration. Proc Natl Acad Sci U S A 106:18809-18814.

Wijesekera LC, Leigh PN (2009) Amyotrophic lateral sclerosis. Orphanet J Rare Dis 4:3.

Wils H, Kleinberger G, Janssens J, Pereson S, Joris G, Cuijt I, Smits V, Ceuterick-de Groote C, Van Broeckhoven C, Kumar-Singh S (2010) TDP-43 transgenic mice develop spastic paralysis and neuronal inclusions characteristic of ALS and frontotemporal lobar degeneration. Proc Natl Acad Sci U S A 107:3858-3863.

Winton MJ, Igaz LM, Wong MM, Kwong LK, Trojanowski JQ, Lee VM (2008) Disturbance of nuclear and cytoplasmic Tar DNA binding protein (TDP-43) induces disease-like redistribution, sequestration and aggregate formation. J Biol Chem 283:13302-13309.

Zhang YJ, Xu YF, Dickey CA, Buratti E, Baralle F, Bailey R, Pickering-Brown S, Dickson D, Petrucelli L (2007) Progranulin mediates caspasedependent cleavage of TAR DNA binding protein-43. J Neurosci 27:10530-10534.

Zhang YJ, Xu YF, Cook C, Gendron TF, Roettges P, Link CD, Lin WL, Tong J, Castanedes-Casey M, Ash P, Gass J, Rangachari V, Buratti E, Baralle F, Golde TE, Dickson DW, Petrucelli L (2009) Aberrant cleavage of TDP-43 enhances aggregation and cellular toxicity. Proc Natl Acad Sci U S A 106:7607-7612.

Zhou H, Huang C, Chen H, Wang D, Landel CP, Xia PY, Bowser R, Liu YJ, Xia XG (2010) Transgenic rat model of neurodegeneration caused by mutation in the TDP gene. PLoS Genet 6:e1000887. 